\title{
Observations on Eimeria species of Dasyprocta leporina (Linnaeus, 1758) (Rodentia: Dasyproctidae) from the state of Pará, North Brazil
}

\author{
Ralph Lainson+ ,Liliane A Carneiro, Fernando T Silveira
}

Departamento de Parasitologia, Instituto Evandro Chagas, Av. Almirante Barrosa 492, 66090-000 Belém, PA, Brasil

Redescriptions are given of the mature oocysts of Eimeria aguti Carini 1935, E. cotiae Carini, 1935 and E. paraensis Carini, 1935, in the faeces of five specimens of the rodent Dasyprocta leporina (Rodentia: Dasyproctidae) from the state of Pará, North Brazil. New information is provided on the sporulation time of these parasites and the prepatent period in experimentally infected D. leporina. Some endogenous stages of $\mathrm{E}$. cotiae are described in the epithelial cells of the ileum, and the absence of any oocysts in the gall-bladder contents of the infected animals indicates that the intestine is also the site of development of E. aguti and E. paraensis. Difficulties in separating E. cotiae and E. paraensis on morphology of the oocysts are discussed. The oocysts of both parasites share many structural features and have a wide size range. It is concluded that although it is at present best to maintain these names, the possibility exists that they were separately given to oocysts of smaller dimensions (E. cotiae) and larger dimensions (E. paraensis) of a single parasite. Location of an endogenous site of development for $\mathrm{E}$. paraensis that is distinctly separate from that of $\mathrm{E}$. cotiae might establish more definitely the separate specific status of the two parasites.

Key words: Dasyprocta leporina - agouti - Eimeria aguti - Eimeria cotiae - Eimeria paraensis - oocysts endogenous stages - Brazil

Following the examination of faeces from a single specimen of an agouti, which he referred to as "Cotia vermelha (Aguti aguti)", Carini (1935) described the mature oocysts of what he considered to be three distinct species of Eimeria, and named them E. paraensis, E. cotiae, and E. aguti. Pellérdy (1974) pointed out that the genus "Cotia" does not exist within the Class Mammalia and listed the host of these parasites as Dasyprocta aguti. This was corrected by Levine and Ivens (1990) to Dasyprocta leporina (Linnaeus, 1758), for which $D$. aguti is now considered to be a synonym (Hussan 1978). The animal was housed in the Parque Zoobotânico of the Museu Emilio Goeldi, in Belém, Pará, North Brazil, and although the exact locality of its origin was not given, it was most likely to have been somewhere in Pará.

We have examined the faeces of $10 \mathrm{D}$. leporina, identified to species by cytogenic analysis, from two different areas of Pará: five of them were passing oocysts of Eimeria species which superficially resembled those described in D. leporina by Carini. In this communication we give re-descriptions of the mature oocysts of E. aguti and those previously described as

Financial support: The Wellcome Trust, London (RL and FTS), Secretaria de Vigilância, Ministério da Saúde, Brasil

Corresponding author: ralphlainson@iec.pa.gov.br

Received 18 October 2006

Accepted 5 February 2007
E. paraensis and E. cotiae (Carini 1935): some endogenous stages, regarded as those of E. cotiae, are described in the ileum. Doubts are raised regarding separation of this parasite and E. paraensis.

\section{MATERIALS AND METHODS}

The agoutis were captured in fruit and vegetable-baited traps. Eight of the animals were from the municipality of Anajás, Island of Marajó, Pará (0.59 S : 49.57 W) and two from the Island of Caratateua (more popularly known as Outero), municipality of Belém, Pará (1.18 S : 48.28 W): they were maintained, in separate cages, on a diet of mixed fruit and vegetables and given water ad libitum. Faecal samples were obtained daily, lightly triturated in $2 \%$ aqueous potassium dichromate solution $\left(\mathrm{K}_{2} \mathrm{Cr}_{2} \mathrm{O}_{7}\right)$, kept at approximately $24^{\circ} \mathrm{C}$ as thin layers in loosely covered Petri dishes, and examined for the presence of coccidial oocysts by both direct microscopical examination and the zinc sulphate flotation method (Baker 1969). Positive samples were examined daily to determine the time required for oocyst sporulation.

In an attempt to locate the site of development of the parasites in D. leporina, two of the naturally infected animals were sacrificed, and a search made for endogenous stages in both the gall-bladder and intestinal epithelium. The entire alimentary tract was fixed in $10 \%$ buffered formalin for subsequent preparation of histological sections of the duodenum, ileum, caecum, and colon. Oocysts and sporocysts were measured using a 100 neofluar objective and $\mathrm{x}$ 10 eyepieces with an ocular micrometer. Photomicrographs were prepared with a Zeiss "Photomicroscope III" and Kodak TMX 100 film. All measurements are given in $\mu \mathrm{m}$ and presented as means, with the range in parentheses, followed by the shape-index (ratio of length/width) and the number measured (n). 
With the object of determining the pre-patent period in newly infected animals, five agoutis with repeatedly negative faeces were force-fed with an aqueous suspension of oocysts, and their faeces subsequently examined daily. Finally, three naturally infected agoutis that had ceased to pass oocysts during a prolonged period in captivity were also fed with the same suspension to see if the animals had acquired immunity to re-infection.

These studies were authorized by Ibama, licence no. 083/2005, Document OF no. 1805/2005/SAHEP/IEC/ SVC MS, Belém, Pará, 10th November, 2005.

\section{RESULTS}

Three of the eight agoutis from Marajó, and both of those from Outeiro, were found to be passing abundant oocysts which were divisible into two distinct morphological groups. Some closely resembled those described by Carini under the name of E. aguti: namely, of small size, spherical to subspherical shape, and with an oocyst wall of a single thin, colourless layer (Figs 1-4). Morphologically, they were readily separated from the second group, the oocysts of which were much larger and possessed a thicker, markedly striated wall of a yellow-brown colour (Figs 6-15). In addition, the sporulation time of $E$. aguti was found to be 5 days, whereas the larger oocysts with the thicker, striated wall required 810 days to complete sporulation.

On oocyst morphology alone, we experienced considerable difficulty in clearly separating the species described as E. cotiae and E. paraensis, due to the wide size range of their oocysts. In addition, morphology of the oocyst wall of some of the parasites suggested that the striated layers had either been lost or had failed to develop (Fig. 11).

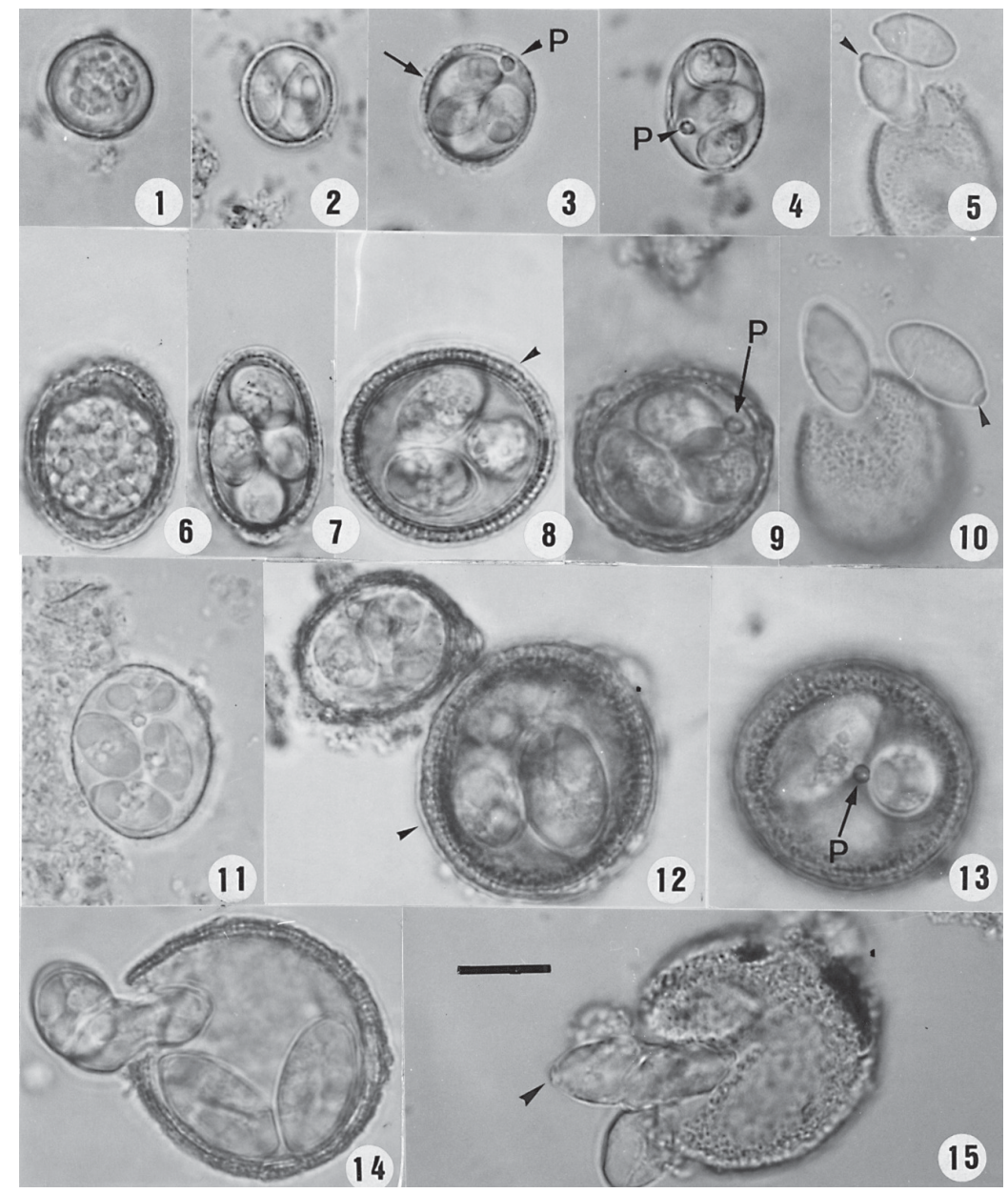

Eimeria species of the agouti, Dasyprocta leporina - Figs 1-5: Eimeria aguti Carini, 1935. Fig 1: immature oocyst. Figs 2-4: intact, mature oocysts showing variation of shape from spherical to broadly ellipsoidal, faint striations of the oocyst wall ( arrow), and presence of a polar body (arrow heads). Fig. 5: crushed and ruptured oocyst showing the tiny Stieda body of two released sporocysts (arrow head) and the faintly discernible pores of the oocyst wall. Figs 6-11: Eimeria cotiae Carini, 1935 - Fig. 6: immature oocyst. Figs 7-9: mature ellipsoidal and spherical oocysts showing the threelayered wall (arrow head) and polar body (P). Fig. 10: crushed oocyst showing two of the four released sporocysts with their small Stieda body (arrow head). Conspicuous pores can be seen in a surface view of the oocyst wall. Fig. 11: loss, or developmental failure, of the thick striated wall layers. Figs 12-15: Eimeria paraensis Carini, 1935 - Figs 12, 13: subspherical and spherical oocysts showing the thick 3-layered wall (arrow head) and polar body (arrow). In Fig. 12 an oocyst of E. cotiae (top, left) is shown for size comparison. Figs 14, 15: crushed oocysts showing the large sporocysts, with a modest Stieda body, and the prominent pores of the oocyst wall in surface view. Compare the sporocyst size of the three species (Figs 5, 10, 14). Bar in Fig. $15=10 \mu \mathrm{m}$ and applies to all other figures. 
No oocysts could be detected in bile removed from the gall-bladder of the two naturally infected animals that were autopsied, suggesting the site of development of the parasites to be in the intestine. Histological sections of the ileum of one of these agoutis revealed abundant macrogamonts and microgamonts in the epithelial cells (Figs 16-19). In size, the macrogamonts and zygotes (young oocysts) were too large to be those of $E$. aguti and too small to be those of E. paraensis: we consequently considered them to be endogenous stages of the parasite regarded as E. cotiae. Meronts were not detected, and no endogenous stages were found in sections of the colon, duodenum or caecum.

The three agoutis that had seemingly recovered from their infections and were challenged by feeding them with oocysts, were all shown to have been re-infected, 8 days later, when oocysts of E. aguti, E. cotiae, and those regarded as E. paraensis reappeared in their faeces.

\section{RE-DESCRIPTIONS}

Eimeria aguti Carini, 1935

(Figs 1-5, 25)

Mature oocysts - Subspherical to spherical, rarely broadly ellipsoidal, $17.8 \times 15.6(11.0 \times 11.0-20.0 \times$ 16.3), shape- index $1.1(1.0-1.3), \mathrm{n}=60$. Oocyst wall a single layer approximately 0.7 thick, smooth, colourless, with no micropyle and only occasionally showing scarcely discernable striations (pores). There is no oocyst residuum but the frequent presence of a round, ovoid or occasionally dumbbell-shaped polar body measuring 1.0 - 2.0 (Figs 3, 4). Sporocysts ovoid, $8.2 \times 5.5(7.4 \times$ 5.2 - $9.6 \times 6.0)$, shape index $1.5(1.4-1.6), \mathrm{n}=38$. There is a very inconspicuous Stieda body, but no sub-Stieda structure. The sporocystic residuum consists of a small mass of fine granules: the sporozoites occupy the full length of the sporocyst but are not notably recurved. No refractile bodies could be detected in them.

Host - The agouti, Dasyprocta leporina (Linnaeus, 1758) (Rodentia: Dasyproctidae).

Site of development - No oocysts were detected in the gall-bladder contents of infected animals, indicating that the parasite's endogenous stages are in some as yet indeterminate part of the gut epithelium.

Endogenous stages - Yet to be described.

Sporulation - 5 days in $2 \%$ potassium dichromate solution at $24^{\circ} \mathrm{C}$.

Pre-patent period - The five agoutis force-fed with oocysts produced faecal pellets containing scanty oocysts on day 7 post-infection, and abundant oocysts on day 8 .

Type locality - Uncertain, but probably some locality in Pará, North Brazil.

Neotype locality - Municipality of Anajás, Island of Marajó, Pará, (0.59 S : 49.57 W)

Type material - No longer available.

Neotype material - Mature oocysts, mixed with those of E. cotiae and those considered to be E. paraensis are preserved in buffered $10 \%$ formaldehyde solution and held in the Department of Parasitology, Instituto Evandro Chagas, Belém, Pará, Brazil, together with phototypes. Depository number 2372.

Prevalence - Five of the 10 D. leporina examined $(50 \%)$ were passing oocysts of E. aguti together with those of E. cotiae and oocysts regarded as E. paraensis.

Pathology - None of the infected animals showed signs of illness and faecal pellets were of normal consistency.

\section{Eimeria cotiae Carini, 1935}

(Figs 6-11, 26)

Mature oocysts - Ovoid to spherical, $21.5 \times 17.5$ (17.0 $\times 14.0-24.0 \times 21.0)$, shape index $1.2(1.1-1.4)$, $\mathrm{n}=50$. Oocyst wall approximately 2.0 , with two distinct inner layers, both of which are striated and of a yellowbrown colour: by bright light microscopy there appears to be a third very fine, colourless outer layer (Fig. 8). There is no micropyle. No oocyst residuum is present, but a spherical to ovoid polar body of about $1.5-2.0$ was seen in approximately $50 \%$ of the oocysts. Sporocysts ovoid, $11.0 \times$ $7.0(9.0 \times 5.2-12.0 \times 7.0)$; shape index $1.7(1.5-1.8), \mathrm{n}=$ 50 . There is a very delicate Stieda body, but apparently no sub-Stieda structure. The sporocyst residuum is a relatively inconspicuous collection of fine granules and globules which may be dispersed or in the form of a more compact, spherical mass. No refractile bodies were detected in the sporozoites. The sporocysts are remarkably constant in their dimensions, and the smallest measurements given here in the range were those made for sporocysts of a single, exceptional oocyst.

Host - The agouti, Dasyprocta leporina (Linnaeus, 1758) (Rodentia: Dasyproctidae).

Site of development - Epithelial cells of the ileum, above the host cell nucleus.

Endogenous stages - As seen in histological sections, spherical to ellipsoidal macrogamonts of approximately $13.0 \times 10.0$ possess a highly vacuolated cytoplasm and an ill-defined nucleus with a small nucleolus (Fig. 17). When mature, the peripheral arrangement of large wall-forming bodies becomes particularly conspicuous (Fig. 18), and the parasite reaches up to 15.0 $\times 13.0$ in size. Its cytoplasm is highly vacuolated and the nucleus very tenuous As seen in Giemsa-stained smears, nearly mature, undivided microgamonts are approximately $19.0 \times 10.0$ (Fig. 20). They produce from 50 to more than 100 microgametes of about $4.0 \times 1.0$, leaving a very prominent residual body of about 14.0-15.0 in diameter (Figs 21, 22). Zygotes are liberated into the gut lumen with the oocyst wall layers only in the initial stage of development (Fig. 19).

Sporulation - At $24^{\circ} \mathrm{C}$, in $2 \%$ aqueous potassium dichromate solution, a few oocysts were mature at 8 days and the majority at 9-10 days.

Prepatent period - 7-8 days.

Type locality - Uncertain: most probably in Pará, North Brazil. 


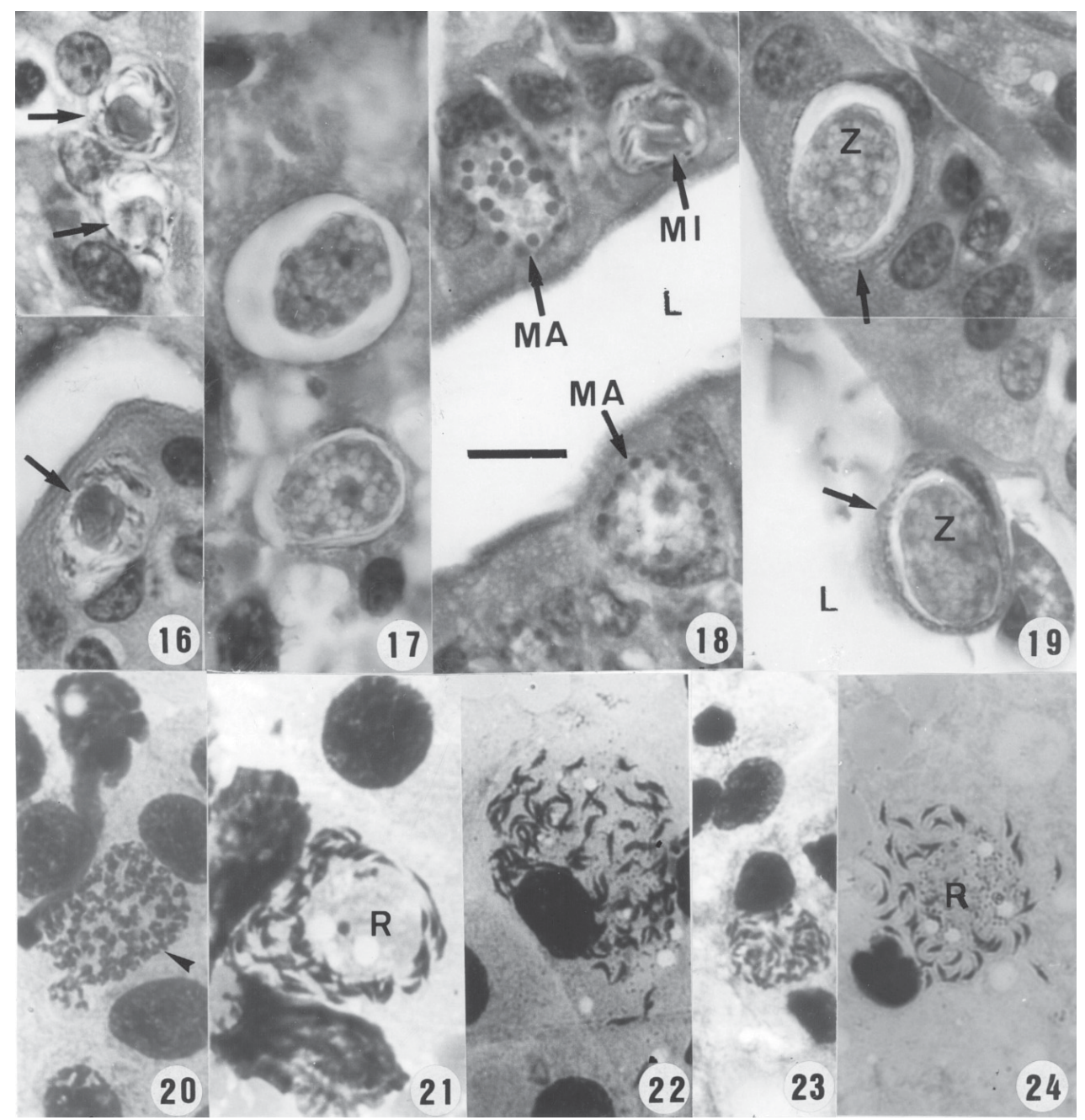

Eimeria species of Dasyprocta leporina. Endogenous stages of Eimeria cotiae in epithelial cells of the ileum. Figs 16-19: histological sections, haematoxylin and eosin staining. Figs 20-24: Giemsa-stained smears - Fig. 16: three mature microgamonts (MI) shedding microgametes (arrowed). Note prominent residual bodies. Fig. 17: macrogamonts (MA) in conspicuous parasitophorous vacuoles: note their highly vacuolated cytoplasm. Fig. 18: MA with prominent wall-forming bodies, and a mature microgamont (MI) shedding microgametes; L: gut lumen. Fig. 19: an intracellular zygote, or young oocyst (top), and another (below) in the process of being shed into the L. Note early development of the striated oocyst wall in both zygotes (arrows). Figs 20-24: MI as seen in Giemsa-stained smears of the ileum epithelium. Fig. 20: almost mature MI. Figs 21, 22: mature MI shedding microgametes. R: large mass of residual cytoplasm. Figs 23, 24: almost mature and mature MI producing much smaller MIs (possibly of $E$. aguti ?). Bar in Fig $18=10 \mu \mathrm{m}$ and applies to all other figures.

Neotype locality - Anajás, Island of Marajó, Pará (0.59 S : $49.57 \mathrm{~W})$.

Type material - No longer available.

Neotype material - Oocysts (mixed with those of $E$. aguti and E. paraensis) and phototypes held in the Department of Parasitology, Instituto Evandro Chagas, Belém. Depository number 2372.

Prevalence - Five of the 10 D. leporina examined were infected, and all were with concomitant infections of E. aguti and E. paraensis.

Pathology - Infected animals showed no signs of illness.

Eimeria paraensis Carini, 1935

(Figs 12-15, 27)

Mature oocysts - Broadly ovoid to subspherical, 26.4 $\times 23.1(24.0 \times 19.2-30.3 \times 28.0)$, shape-index $1.1(1.0-$ $1.4), n=46$. The oocyst wall, averages approximately 2.5 in thickness and has no micropyle. By bright light microscopy, three layers are visible: an outer smooth, very thin and colourless membrane, and two inner, thicker, yellow-brown and markedly striated layers (Fig. 12). No oocyst residuum, but a conspicuous polar body of up to $2.6 \times 2.2$ was visible in approximately $50 \%$ of the oocysts (Fig. 13). Sporocysts ovoid, $15.5 \times 8.3(14.6 \times$ $7.4-16.3 \times 8.0)$, shape-index $1.9(1.5-2.2), \mathrm{n}=32$. There is a modest Stieda body (Fig.15), but no apparent sub-Stieda structure. The sporocyst residuum is in the form of a loosely disposed mass of fine granules and small globules. No refractile bodies were visible in the sporozoites, which occupy the full length of the sporocyst and are moderately recurved at their ends. 

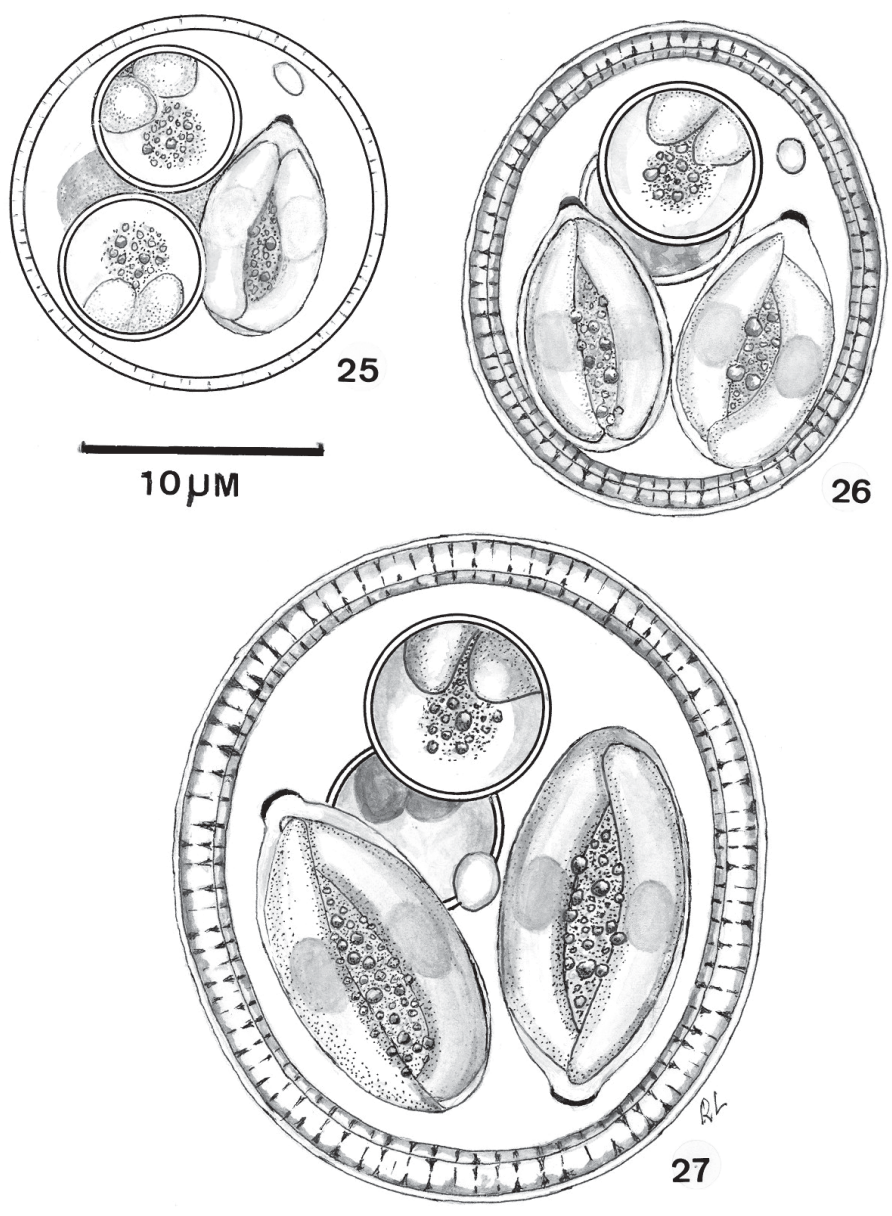

Figs 25-27: mature oocysts of some Eimeria species of the agouti, Dasyprocta leporina - Fig. 25: E. aguti Carini, 1935. Fig. 26: E. cotiae Carini, 1935. Fig. 27: E. paraensis Carini, 1935.

Host - The agouti, Dasyprocta leporina (Linnaeus, 1758) (Rodentia: Dasyproctidae).

Site of development - In some as yet indeterminate part of the intestine

Endogenous stages - Yet to be described.

Sporulation - A few oocysts were mature after 8 days in $2 \%$ potassium dichromate at approximately $24^{\circ} \mathrm{C}$, with the majority fully mature after 9-10 days.

Prepatent period - 7-8 days.

Type locality - Uncertain, but most probably in Pará, North Brazil.

Neotype locality - Anajás, Island of Marajó, Pará, North Brazil (0.59 S : $49.57 \mathrm{~W})$.

Type material - No longer available.

Neotype material - Oocysts (mixed with those of $E$. aguti and E. cotiae) and phototypes in the Department of Parasitology of the Instituto Evandro Chagas, Belém. Depository number 2372.

Prevalence - 5 of the $10 \mathrm{D}$. leporina examined were infected, and all were with concomitant infections of $E$. aguti and E. cotiae.
Pathology - Infected animals showed no signs of illness and produced faecal pellets of normal consistency.

\section{DISCUSSION}

Of the enormous number of eimeriid coccidia so far named, most descriptions have included only the morphology of the sporulated oocysts and, in naming new species, it has become customary to regard morphologically identical oocysts in closely related hosts as conspecific, while morphologically similar oocysts in taxonomically widely separated hosts are of different species: finally, and more importantly in our present study, it is generally assumed that morphologically different oocysts in the same host also represent different species.

As the oocysts of E. paraensis, E. cotiae, and E. aguti were first described in D. leporina, it was reasonable to suppose that we were dealing with the same species of Eimeria in the same animal from the same geographic area. It is unfortunate, however, that based on the generally accepted and present-day requirements for the description of coccidial oocysts (Duszynski \& Wilber, 1997), Carini's descriptions are somewhat inadequate, and this makes a comparison difficult. Thus, there is no indication of the number of oocysts and sporocysts that were measured for each parasite and, in 
some cases (E. cotiae and E. aguti), no indication of their size range.

We have no doubt regarding the identification of $E$. aguti, as the morphology of the mature oocyst given in our re-description closely matches that described by Carini: namely, oocysts $17.8 \times 15.6$ versus $16.0-17.0$ diameter and sporocysts $8.2 \times 5.5$ versus $10.0 \times 6.0$. There is agreement, too, regarding the inconspicuous Stieda body of the sporocyst and the lack of a sub-Stieda body, and the singlelayered, colourless wall of the oocyst: Carini's failure to figure a polar body in oocysts of this species (or those of E. cotiae and E. paraensis) was possibly because he studied insufficient oocysts. We detected them in only approximately $50 \%$ of the oocysts: polar bodies lying beneath the sporocysts will be most difficult to detect. We found no endogenous stages that we could confidently ascribe to $E$. aguti. In Giemsa-stained smears of the ileum containing developing and mature microgamonts of $E$. cotiae (Figs 20-22), however, we found some such stages producing considerably smaller microgametes (Figs 23, 24). The small size of these suggested that they might be those of E. aguti, and that development of this parasite is also in the ileum.

Our separation of E. cotiae and E. paraensis, was far from easy in all five infected agoutis because, from our observations, their oocysts share many characteristics. These include the same sporulation time of 8-10 days and the same pre-patent period of 7-8 days; ovoid to subspherical oocysts with a shape-index of 1.2 and 1.1 respectively; a thick, striated and multilayered oocyst wall; similarly shaped sporocysts; and a single polar body in about $50 \%$ of the oocysts. On the assumption that the smaller oocysts of this type are E. cotiae, the only morphological difference shown in our study of these two parasites is really one of size, with E. cotiae appearing as a scaled-down version of E. paraensis. Carini's brief description gives the impression that the oocysts of E. cotiae are consistently ovoid and with an average of $29.0 \times 18.0$, which gives a shape-index of 1.6. We, however, found a great variation of shape from ovoid to subspherical (Figs 7, 8), and our mean for 50 oocysts measured was $21.5 \times 17.5$, giving a shape-index of only 1.2. In addition, we considered the oocyst wall to normally have two distinct inner, striated layers and a third outer, very fine and colourless membrane: Carini figured the oocyst wall as a single striated layer.

In the original description, measurements of the oocysts of E. paraensis were given as 33.0-40.0 $\times 30.0$ 35.0 and the oocyst wall was described as being 2.0 thick and composed of two yellow-brown, striated layers: no polar body was figured. Measurements of the sporocysts were given as $20.0 \times 11.0$, and the Stieda body was figured as being very inconspicuous. In the five infected agoutis we studied none of the oocysts we considered as E. paraensis exceeded $30.3 \times 28.0$, and the oocyst wall, like that of $E$. cotiae, seemed to be composed of two inner, yellow-brown and striated layers and a very fine and colourless outer membrane (Figs 12,14). A polar body averaging $2.6 \times 3.0$ was present in about $50 \%$ of the oocysts (Fig. 13). The sporocysts averaged only $15.5 \times 8.3$ and, like those figured by Carini, possessed a small Stieda body.
In conclusion, although there is some similarity in Carini's description of the basic morphology of the oocysts and sporocysts of E. cotiae and E. paraensis and that we give in the present study, our measurements differ considerably. We feel it best at present, however, to maintain these specific names, but with the reservation that they may have been given to the extremes in oocyst/ sporocyst size-range of a single parasite. In this respect it is notable that although there is usually a consistent and moderate size range for the oocysts of a given species, there are instances when there may be a very great variation in either the length or width. The mature oocysts of E. damnosa of the squirrel Sciurus spadiceus, for example, range from $18.0 \times 15.0-40.2 \times 30.0$ (Lainson et al. 2005). In addition, there may be striking differences in the structure of the wall of the oocysts of a given species in the same host. The oocyst wall of $E$. bragancaensis, in the bat Peropteryx macrotis, is normally two layered and with the outer layer prominently striated and yellow-brown in colour. This layer may be lost (or fail to develop), however, leaving the oocyst with the thin, colourless and non-striated inner layer and, therefore, a very different appearance (Lainson \& Naiff 2000). Similar variation in wall structure has been reported for E. auburnensis (Christensen \& Porter 1939) and E. robusta (Supperer \& Kutzer 1961).

So far we have been able to confidently detect the endogenous stages of only one parasite in the ileum and, from the size of the macrogamonts and zygotes, we have concluded that these belong to E. cotiae. The possibility that the developmental stages of E. aguti are also in the ileum, however, has already been discussed. No endogenous stages were encountered in the caecum, colon or duodenum of the same animal, but it is to be admitted that this could well be due to the limited amount of these parts of the intestine it has been possible to examine. It is hoped that continuing examination of sections from all parts of the intestine of infected animals will eventually locate undoubted endogenous stages of $E$. aguti and, at the same time, that location of endogenous stages of $E$. paraensis will establish more definitely the separate specific status of this parasite and E. cotiae.

Rose (1973) discussed, at length, acquired immunity among the coccidia as a result of active infection. There would appear to be a considerable variation in the capacity of different coccidian species to elicit this in the same host species, and although an immune response occurs in most species, there are records of very slight or no acquired immunity following active infection, in the case of some others. Thus, Todd and Hammond (1968) found that repeated experimental infections could be produced with. E. callospermophili in the ground squirrels Spermophilus armatus, S. richardsoni, and $S$. variegatus, while Ernst et al. (1968) reported repeated reinfections by E. utahensis in the kangaroo rats, Dipodomys ordii and D. microps. Versényi and Pellérdy (1970) considered the immune response of geese to infection with $E$. anseris as very weak and incapable of preventing reinfection of these birds.

We are confident that we obtained experimental reinfections with E. aguti, E. cotiae, and the parasite 
regarded as E. paraensis in the three agoutis that had recovered from natural infections. They had shown no oocysts in their faeces for several weeks and the prepatent period following challenge was 7-8 days - exactly that seen in five other experimentally infected D. leporina.

\section{ACKNOWLEDGEMENTS}

To Dr Manoel do Carmo Pereira Soares, Hepatology Department, Instituto Evandro Chagas who kindly made the eight specimens of $D$. leporina from Anajás available for our examination, and to Dr Nelson AB Ribeiro, of the same Department, for his cytogenic identification of all the animals. Constância M Franco, Manoel C de Souza, and Antonio J de O Monteiro, Department of Parasitology, IEC provided their excellent technical assistance.

\section{REFERENCES}

Baker JR 1969. Parasitic Protozoa, Hutchinson \& Co., London.

Carini A 1935. Sur trois nouvelles Eimeria de Cotia vermelha. Ann Parasit Hum Comp13: 342-344.

Christensen JF, Porter DA 1939. A new species of coccidium from cattle, with observations on its life history. Proc Helm Soc Wash 6: 45-48.

Duszynski DW, Wilber PG 1997. A guideline for the preparation of species descriptions in the Eimeriidae. J Parasitol 83: 333-336.

Ernst JV, Hammond DM, Chobotar 1968. Eimeria utahensis sp.n. from kangaroo rats (Dipodomys ordii and D. microps) in northwestern Utah. J Protozool 15: 430-432.
Hussan AM 1978. The Mammals of Suriname, Brill, Leiden, Netherlands, $569 \mathrm{pp}$

Lainson R, Naiff RD 2000. On Eimeria bragancaensis n.sp. (Apicomplexa: Eimeriidae) and tissue cysts of an unidentified protozoan in the bat Peropteryx macrotis (Chiroptera:Emballonuridae) from Amazonian Brazil. Parasite 7: 123-129.

Lainson R, Brigido MCO, Silveira FT 2005. Parasites of the squirrel Sciurus spadiceus (Rodentia: Sciuridae) from Amazonian Brazil, with particular reference to Eimeria damnosa n.sp. (Apicomplexa: Eimeriidae). Parasite 12: 305-315.

Levine ND, Ivens V 1990. The Coccidian Parasites of Rodents, CRC Press, Boca Raton, FL, 228 pp.

Pellérdy L 1974. Coccidia and Coccidiosis, Verlag Paul Parey, Berlin, Hamburg, 959 pp.

Rose ME 1973. Immunity. In DM Hammond, PL Long (eds), The Coccidia, University Park Press, Baltimore and Butterworths, London, p. 295-341.

Supperer R, Kutzer E 1960-1961. Die Kokzidien von Reh, Hirsch und Gemse. Jub Jahrbuch ÖAFW: 128-136.

Todd KS, Hammond DM 1968. Life cycle and host specificity of Eimeria callospermophili Henry, 1932 from the Uinta ground squirrel Spermophilus armatus. J Protozool 15: 1-8.

Versényi L, Pellérdy L 1970. Pathological and immunological investigations of the anseris-coccidiosis of the domestic goose (Anser anser dom.). Acta Vet Acad SciHung 20: 103-107. 
